[0212-7199 (2006) 23: 4; pp 166-172] ANALES DE MEDICINA INTERNA Copyright (C) 2006 ARAN EDICIONES, S.L.

AN. MED. InTERNA (Madrid) Vol. 23, N. $^{\circ} 4$, pp. $166-172,2006$

\title{
Estudio de la población hipertensa muy anciana ingresada en un hospital comarcal
}

\author{
M. J. NÚÑEZ FERNÁNDEZ, J. PAZ ESQUETE ${ }^{1}$, B. CASTRO PAREDES, \\ J. C. GARCÍA GARCÍA, F. J. FRAILE AMADOR, A. LÓPEZ SOTO \\ Unidad de Medicina Interna. Fundación Pública Hospital Comarcal do Salnés. \\ Vilagarcía de Arousa. Pontevedra. ${ }^{~}$ Servicio de Medicina Preventiva. Complejo \\ Hospitalario de Pontevedra
}

STUDY OF HYPERTENSIVE VERY ELDERLY POPULATION ADMITTED TO A REGIONAL HOSPITAL

\section{RESUMEN}

Objetivos: Conocer las características de la población hipertensa muy anciana (edad igual o superior a 80 años) ingresada en nuestro centro hospitalario.

Material y métodos: Estudio prospectivo, incluyendo a pacientes con hipertensión (HTA) ingresados en nuestro centro. Se estudiaron: parámetros demográficos, clínicos, factores de riesgo vascular, medidas higiénico-dietéticas, tratamiento farmacológico, causa del ingreso, y determinaciones bioquímicas

Resultados: Se incluyeron 92 pacientes (71 mujeres); representan el $14 \%$ de los ingresos. Dos terceras partes no habían realizado estudios académicos; siendo el $60 \%$ de procedencia rural. Casi la mitad $(41 \%)$ eran diabéticos tipo II y un $27 \%$ tenía dislipemia. El 62\% tenían 4 o mas, factores de riesgo cardiovascular. El ingreso hospitalario se relacionó con la HTA en la mitad de los casos, y un $61 \%$ ya había tenido previamente algún evento cardiovascular. Las medidas higiénico-dietéticas mas frecuentes son: no fumar (95\%), no alcohol (81\%), y dieta sin sal (75\%). Los fármacos más usados son: diuréticos e IECAs. La mortalidad global fue dos veces superior a la de los hipertensos de menos de 80 años.

Concluciones: Los hipertensos muy ancianos ingresados en nuestro centro, son fundamentalmente mujeres, de procedencia rural y sin estudios académicos. Dicho ingreso es atribuible directamente a la HTA en la mitad de los casos. Son una población de alto riesgo cardiovascular, con eventos previos cardíacos y cerebro-vasculares. Confiesan realizar las medidas higiénico-dietéticas recomendadas. Los diuréticos son los fármacos mas utilizados para la HTA. Como era de esperar la mortalidad en este grupo es muy alta.

PALABRAS CLAVE: Hipertensión. Población muy anciana. Diabetes. Epidemiología.

\section{ABSTRACT}

Objectives: To analyse the characteristics of the hospitalized very elderly people (age equal or superior to 80 years) with hypertension (HT).

Patients and methods: Prospective study including the patients with $H T$ who need hospitalization in our medical institution. Data collected were: demographic, clinical parameters, factors of vascular risk, hygienic - dietetic strategies, pharmacological treatment, cause of hospitalization, and biochemical determinations.

Results: There were included 92 very elderly patients ( 71 women); they represent $14 \%$ of total hospitalized people. Two third parts had not realized academic studies, being $60 \%$ of rural origin. Almost the half (41\%) was diabetic, and $27 \%$ had dyslipidemia. Overall $62 \%$ had 4 or more factors of cardiovascular risk. The hospitalization was related to the HT in the half of the cases, and $61 \%$ had already previously some cardiovascular event. The lifestyle modifications more frequent were: not to smoke (95\%), not alcohol (81\%), and diet without salt (75\%). Diuretics were the most frequent anti-hypertensive agent used. The global mortality was two times superior to the hypertensive population $<80$ years in the same period.

Conclusions: The very elderly hypertensive patients of our study are fundamentally women, of rural origin and without academic studies. The above mentioned hospitalization is attributable directly to the HT in the half of the cases. They are a population of high cardiovascular risk, with previous events cardiac and cerebral-vascular. They confess to realize frequently the hygienic - dietetic strategies recommended. The diuretics are the anti-hypertensive agents most used for the HT. Since it was of waiting for the mortality in this group it is high.

KEY WORDS: Hypertension. Very elderly people. Diabetes. Epidemilogy

Núñez Fernández MJ, Paz Esquete J, Castro Paredes B, García García JC, Fraile Amador FJ, López Soto A. Estudio de la población hipertensa muy anciana ingresada en un hospital comarcal. An Med Interna (Madrid) 2006; 23: 166-172.

\section{INTRODUCCIÓN}

La hipertensión arterial (HTA) representa desde hace años un problema sanitario de extraordinaria magnitud, tanto en países desarrollados como en aquellos en vías de desarrollo (1). Aunque la detección de HTA es variable a lo largo de los diferentes países, si es constante su alta prevalencia en personas mayores de 60 años, afectando entre $60-70 \%$ de dicha población $(1,2)$.

La tendencia demográfica actual en países occidentales se dirige hacia un incremento de la población anciana. Nuestra 
Comunidad Autónoma es también un claro exponente de esta tendencia, así en los últimos 25 años (1975-1999), la población gallega mayor de 64 años, se incrementó un 58\% (Datos de la Xunta de Galicia; www.ige.xunta.es). Dentro de este grupo las personas mayores de 80 años, son el segmento poblacional que mas rápidamente está creciendo (3). Hace años diferentes estudios epidemiológicos han demostrado la relación existente entre hipertensión, edad avanzada y presencia de eventos cardiovasculares (4). Sin embargo los ancianos hipertensos y sobre todo los mayores de 80 años, siempre fueron un grupo poblacional en cierto modo olvidado, a pesar de que por definición son unos pacientes de muy alto riesgo cardiovascular (RCV) (5).

Últimamente existe un renovado interés por parte de la comunidad médica en los hipertensos muy ancianos tal como lo demuestran las recientes publicaciones $(6,7,8,9)$, así como los estudios puestos en marcha, cuyos resultados aclararan aspectos específicos sobre estos pacientes (10).

Pretendemos con el presente trabajo realizar un estudio descriptivo en una población hipertensa muy anciana, seleccionada a través de su ingreso en nuestro centro hospitalario. Se realiza el análisis de toda una serie de características, que van desde datos demográficos, epidemiológicos, clínicos, y conductales. El grupo seleccionado es comparado con un grupo de hipertensos ingresados en el mismo período, pero de edad inferior a 80 años.

\section{MATERIAL Y MÉTODOS}

Se realiza un estudio descriptivo transversal sobre pacientes hipertensos muy ancianos ingresados en la Unidad de Medicina Interna de nuestro centro hospitalario.

1. Centro sanitario. La Fundación Pública Hospital Comarcal do Salnés, es un centro sanitario de reciente apertura (septiembre de 2001), atiende a una población de unos 70.000 habitantes, pertenecientes a 7 ayuntamientos de la zona noroeste de la provincia de Pontevedra. Consta de 80 camas, de las cuales, la mitad dependen de la Unidad de Medicina Interna. En dicha Unidad trabajan lo componentes del equipo investigador del presente trabajo. El resto de las camas dependen de especialidades Quirúrgicas y de Pediatria. No existen en el centro Unidad de Cuidados Intensivos ni Unidad de Coronarias.

2. Población a estudio. Se incluyeron de forma prospectiva en el estudio, los pacientes con edad superior o igual a 80 años, con antecedentes conocidos de HTA esencial (HTA sistólica asilada, HTA diastólica aislada, o HTA sistólica-diastólica), de mas de 1 año de evolución, ingresados en nuestra Unidad, con independencia de la causa que motivase el ingreso.

3. Duración del estudio. Fue realizado durante un total de 7 meses, de forma discontinua, iniciándose en noviembre de 2002, y terminando en julio de 2003, siempre con duración mínima de períodos mensuales.

4. Datos recogidos. En las primeras 24-48 horas del ingreso, los componentes de nuestra Unidad pasaron un cuestionario contestado mayoritariamente por los pacientes y cuando esto no era posible, era completado por los familiares. Se recogieron diferentes datos demográficos: edad, sexo, años de evolución de la hipertensión, facultativo que estableció el diagnóstico de hipertensión, niveles de estudios académicos, área de procedencia.

5. Factores de riesgo cardiovascular: los aceptados por la Sociedad Europea de Hipertensión/Sociedad Europea de Car- diología (2003) (11): tabaquismo (consumo de al menos 1-2 cigarrillos al día), diabetes (glucosa basal superior a 126 mgr/dl, o postpandrial superior a $198 \mathrm{mgr} / \mathrm{dl}$, en situación basal; o uso de antidiabéticos orales y/o insulina), edad (varones superior a 55 años; hembras superior a 65 años), dislipemia (colesterol total superior a $250 \mathrm{mgr} / \mathrm{dl}$, o LDL superior a $155 \mathrm{mgr} / \mathrm{dl}$, o HDL inferior a $40 \mathrm{mgr} / \mathrm{dl}$ en varones o inferior a $50 \mathrm{mgr} / \mathrm{dl}$, en hembras; o uso de hipolipemiantes por diagnóstico previo de dislipemia), antecedentes familiares de enfermedad cardiovascular precoz (en varones antes de los 55 años, y en mujeres antes de los 65 años).

6. Afectación de órganos diana: hipertrofia ventricular izquierda en ECG (Sokolow-Lyons $>38 \mathrm{~mm}$ ) o en ecocardiograma (IMVI: $\mathrm{V} \geq 125, \mathrm{M} \geq 110 \mathrm{~g} / \mathrm{m}^{2}$ ). Demostración ecográfica de engrosamiento de la pared arterial o placas de aterosclerosis. Elevación ligera de la creatinina (V 1,3-1,5, M 1,2-1,4 mg/dl). Microalbuminuria (30-300 mg/24 h).

7. Condiciones clínicas asociadas: enfermedad cerebrovascular: ictus isquémico, hemorragia cerebral, ataque isquémico transitorio. Cardiopatía: infarto de miocardio, angina, revascularización coronaria, insuficiencia cardiaca congestiva. Nefropatía: nefropatía diabética, proteinuria (> $300 \mathrm{mg} / 24$ h) o insuficiencia renal (creatinina $\mathrm{V}>1,5, \mathrm{M}>1,4 \mathrm{mg} / \mathrm{dl}$ ). Arteriopatía periférica. Retinopatía avanzada: hemorragias, exudados, edema de papila

8. Realización de pruebas complementarias antes de su ingreso en nuestro centro: ecodoppler de troncos supra-aórticos, ecocardiografía transtorácica, ecografía renal y fondo de ojo.

9. Determinación de la causa del ingreso y de su relación con la HTA (entendidos como tales aquellos ingresos cuya causa fueron: fallo cardíaco, accidente cerebrovascular agudo no cardioembólico, accidente isquémico transitorio y hemorragia cerebral).

10. Evolución clínica: se siguió la evolución del paciente desde su ingreso en nuestro centro hasta su alta clínica, fallecimiento o traslado a otro centro, analizándose el cálculo de estancia. No se han incluido en el grupo de fallecimientos los existentes en el grupo de traslados

11. Medidas higiénico dietéticas: se considera dieta baja en sal (cuando es inferior aproximadamente a $6 \mathrm{~g}$ de cloruro sódico diarios), dieta rica en vegetales (consumo de mas de cuatro días de vegetales en el plato principal del almuerzo o cena) abstinencia de tabaco (cuando dicho hábito se interrumpió hace mas de 5 años, previo a este ingreso), dieta baja en grasas (cuando el consumo de grasas saturadas no es superior a 1 día a la semana en el plato principal del almuerzo o cena), abstinencia de alcohol (no consumo de alcohol de forma diaria en los últimos dos años).

12. Encuesta. A todos aquellos pacientes sin limitaciones (entiéndase afasia, demencia, u otras causas de tipo orgánico o psíquico que impidiesen la contestación a las preguntas de dicha encuesta), se solicitó contestasen a dos preguntas: ¿Conoce algún tipo de complicación grave para la salud que la hipertensión pueda producir? ¿Conoce los valores de TA por encima de los cuales se considera HTA?

13. Estratificación del riesgo en tres grupos: a) sin factores de riesgo; b) con mas de un factor de riesgo, pero sin lesión de órganos diana (LOD), ni estados clínicos asociados (ECA), ni diabetes; y c) con LOD, ECA o diabetes (12)

14. Tratamiento farmacológico: se recogió en la historia clínica al ingreso el tratamiento farmacológico según las familias de fármacos, y al finalizar la estancia se procedió de igual forma según el informe clínico de alta. 
15. Comparación entre poblaciones hipertensas ingresadas en nuestra Unidad de Medicina Interna: población con edad igual o superior a 80 años, y población con edad inferior a 80 años.

\section{ANÁLISIS ESTADÍSTICO}

\section{ESTUDIO DESCRIPTIVO}

Las variables cualitativas se expresan como porcentajes y frecuencias absolutas Las variables numéricas se expresan con la media y la desviación típica cuando seguían distribución normal, o con la mediana y el rango intercuartílico cuando la distribución resultó ser no gaussiana. Como pruebas de normalidad se emplearon el test de Kolmogorov-Smirnov y el de Shapiro-Wilk.

\section{ANÁLISIS UNIVARIANTE}

La comparación de las variables categóricas se llevó a cabo mediante la prueba c 2 o el test exacto de Fisher. Al comparar los diferentes grupos de estudio, para el análisis de variables continuas, se emplearon la prueba T-student, cuando seguían distribución normal, o U de Mann-Whitney cuando la distribución resultó no normal. Para valorar la posible correlación entre variables continuas se utilizaron el coeficiente de correlación de Pearson cuando resultaban gaussianas y Rho de Spearman cuando no lo eran. Se consideró estadísticamente significativa una $\mathrm{p}<0,05$.

Los análisis se realizaron con el programa Statistical Package for Social Sciencies (SPSS), versión 12.0.1 en castellano

\section{RESULTADOS}

Se recogieron de forma correcta un total de 199 encuestas a 174 pacientes hipertensos con edad superior a 15 años (hubo 25 reingresos), dentro de este grupo existían 92 pacientes hipertensos con edad igual o superior a 80 años, que representan el $52 \%$

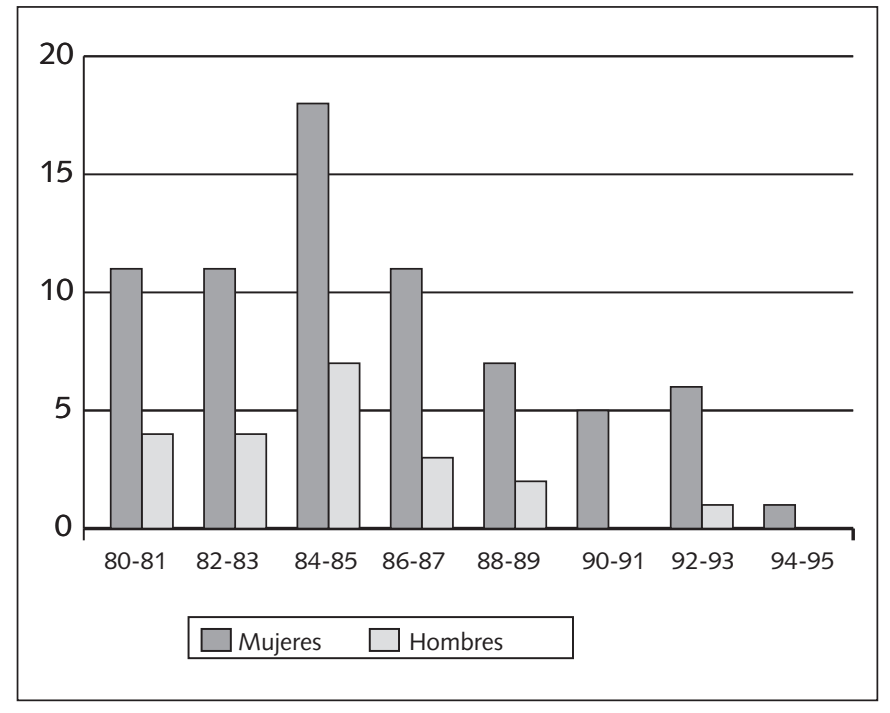

Fig. 1. Sexo y edad.

de los hipertensos ingresados, y el 14\% de todos los ingresos en el área de Medicina Interna durante el periodo de estudio. Había 71 mujeres y 21 varones. La edad media fue de 85 años, con un rango entre 80 y 95 años (Fig. 1). El diagnóstico de HTA fue establecido en primer lugar por los médicos de familia (67\%), seguidos por los especialistas de atención hospitalaria $(7,6 \%)$.

La evolución en años de la HTA fue: 1-5 años: 19\%; 6-10 años: $33 \%$; $11-15$ años: $12 \%$; mas de 15 años: $12 \%$. Los niveles de estudios académicos realizados por la población estudiada fue: ninguno $66 \%$, estudios básicos $7 \%$, bachiller $3 \%$. El $60 \%$ procedía del área rural.

Además de la HTA y la edad, otros factores de riesgo cardiovascular encontrados fueron: la diabetes (41\%), y la dislipemia (27\%). El número total de factores de riesgo cardiovascular fue: Tres, en 26\%. Cuatro, en 34\%. Cinco o más de cinco, en $28 \%$ (Fig. 2).

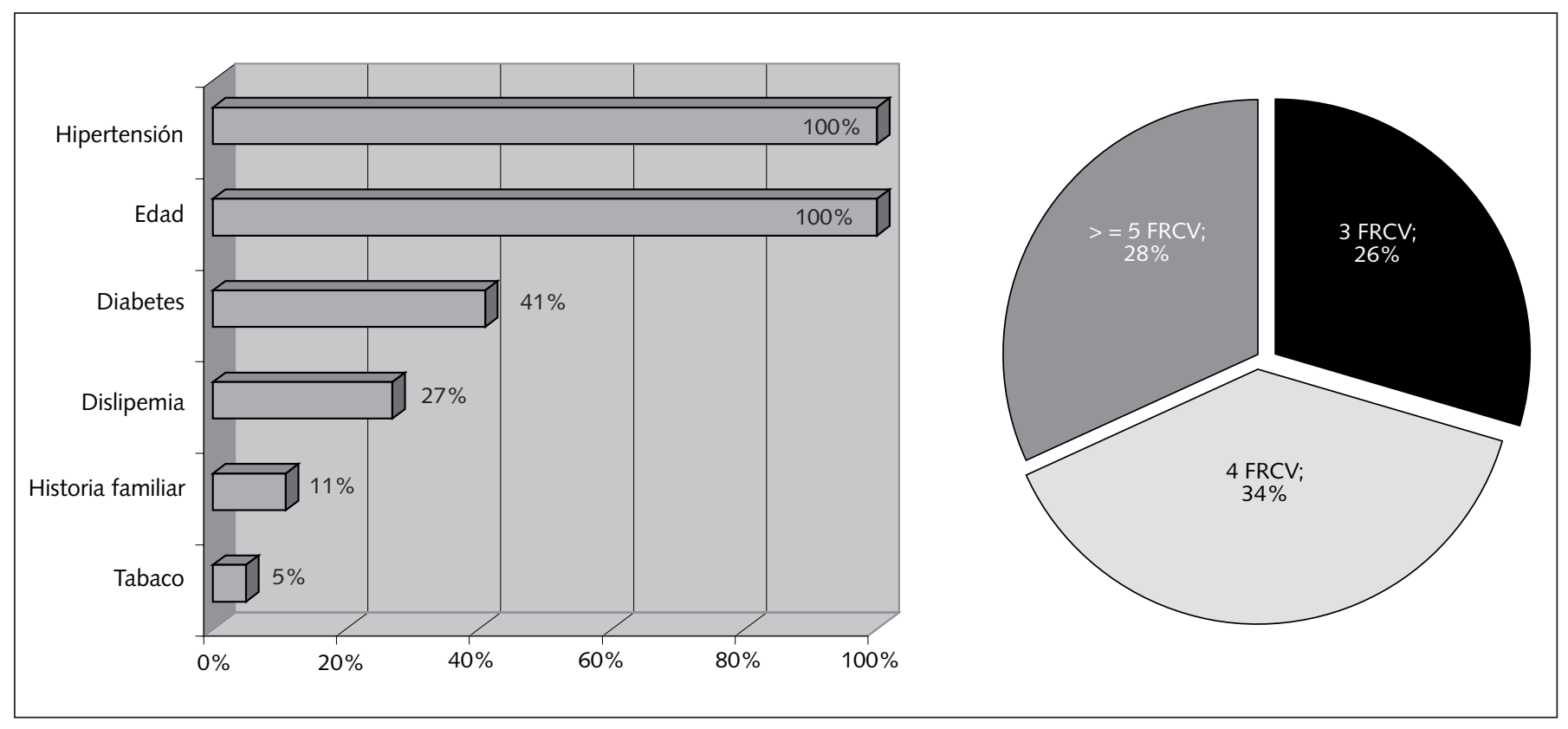

Fig. 2. Estudio de los factores de riesgo cardiovascular (FRCV). 
La distribución de pacientes siguiendo la estratificación del riesgo cardiovascular en las tres categoría conocidas, se estableció de la siguiente forma: Categoría A: 0\%. Categoría B: $18 \%$. Categoría C: $82 \%$.

Se documentaron las siguientes condiciones clínicas asociadas: cardiopatía $32 \%$, enfermedad cerebro-vascular $32 \%$, nefropatía $15 \%$, enfermedad vascular periférica $6 \%$, y retinopatía hipertensiva $2 \%$. La afectación de órganos diana referida o encontrada fue: HVI 24\%, afectación renal 16\%. Las pruebas complementarias realizadas con anterioridad a su ingreso fueron: ecocardiografía transtorácica en $29 \%$, estudio de fondo de ojo en $19 \% \%$, y ecografía renal en $10 \%$,

Se estudiaron las causas del ingreso y se establecieron como relacionados de forma directa con la HTA en 56\%. Los principales diagnósticos codificados en este apartado fueron: insuficiencia cardíaca $(31 \%)$ y enfermedad cerebro-vascular aguda $(25 \%)$.La presencia de eventos cardiovasculares previos al ingreso se confirmó en el $60 \%$ de los pacientes. La evolución clínica fue hacia la curación y alta hospitalaria en $80 \%$, traslado a otro centro hospitalario en $11 \% \mathrm{y}$, fallecimiento en $8,7 \%$. La estancia media de los pacientes fue de 11,8 días, con un rango comprendido entre 1-41 días.

De las medidas higiénico dietéticas se realizaban las siguientes: abstinencia de tabaquismo (95\%), abstinencia de alcohol $(81 \%)$, dieta baja o sin sal $(75 \%)$, dieta baja e grasas $(55 \%)$, y rica en verduras $(47 \%)$. Existía una persona que no hacían ninguna medida higiénico dietética; el 23\% hacía 1 o 2; el 75\% hacía 3 o mas de 3 medidas (Fig. 3)

Con relación al tratamiento farmacológico antihipertensivo, los fármacos usados fueron: diuréticos (52\%); IECAs (43\%); ACC (24\%); ARA II (14\%); beta bloqueantes (4\%). En el $4 \%$ de los pacientes no fue posible conocer los fármacos que tomaban. La combinación de fármacos antihipertensivos se distribuyó como sigue: el $45 \%$ tomaba 1 fármaco. El $45 \%$ tomaba 2 o más de 2 fármacos. Según el número total de fármacos que el paciente tomaba a su ingreso, se constató lo siguiente: el $53 \%$ de los pacientes fueron alta con el mismo número de fármacos que a su ingreso. Fue preciso un incremento en dicho número al alta en el $24 \%$, precisando disminución en el $22 \%$ (Fig. 4)

La encuesta pasada a la población hipertensa arrojó los siguientes resultados: el $88 \%$ no conoce ningún valor límite de la HTA, ni tensión sistólica ni diastólica; el 12\% conoce un único valor límite. De la segunda pregunta, el $84 \%$ reconocían

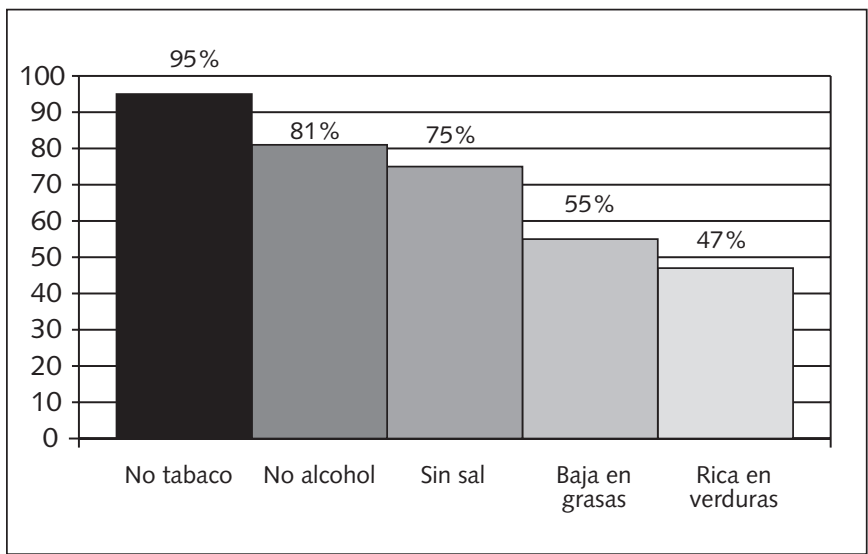

Fig. 3. Medidas higiénico dietéticas utilizadas. que la HTA podía provocar un problema importante de salud, incluida la muerte. En 48 pacientes de la serie no se pudo realizar la encuesta al existir limitaciones para su contestación (demencia, accidente cerebrovascular agudo o remoto).

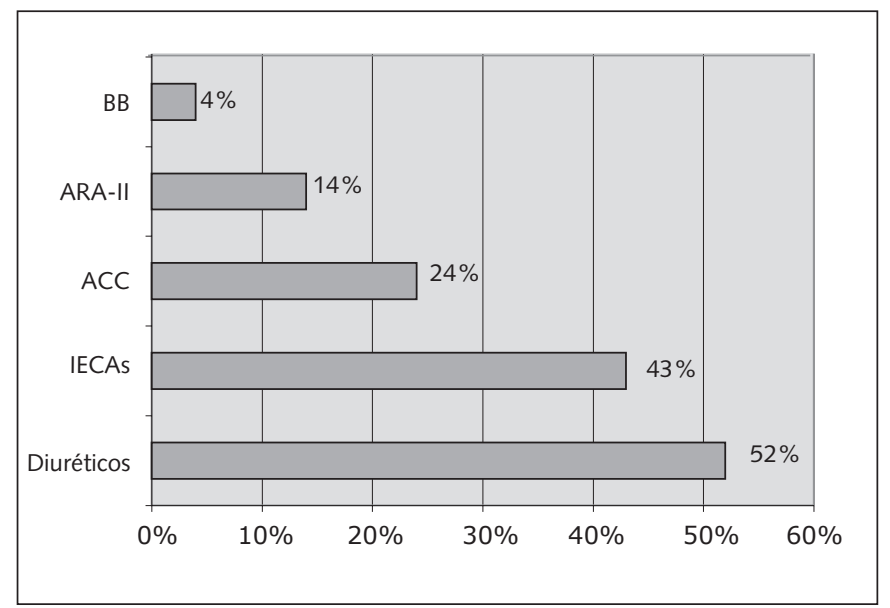

Fig. 4. Tratamiento farmacológico anti-hipertensivo. Abreviaturas: BB: beta-bloqueantes. ARA-II: antagonistas de receptor de angiotensina II. ACC: antagonistas de canales del calcio. IECAs: inhibidores de enzima convertidor de angiotensina.

La comparación entre las dos poblaciones así como los resultados de las determinaciones analíticas se especifican en la Tabla I.

\section{DISCUSIÓN}

Existe en nuestra serie un claro predominio del sexo femenino en la población estudiada, dato coincidente con los aportados por estudios epidemiológicos similares al nuestro y con mayor número de casos $(6,8,9,13)$; la explicación se encuentra en la relación ya conocida entre edad e hipertensión: cuanta mas edad, mas prevalencia de hipertensión (14), y por ser las mujeres mas longevas y las predominantes en los últimos segmentos de edad en la pirámide poblacional.

Otra de las características notorias y que resalta en la serie, es la mayor presencia de personas del entorno rural. Quizás no es de extrañar este dato, al existir un claro componente rural en la población dependiente de la asistencia sanitaria en nuestro centro. Algunos autores justifican también este predominio en la existencia de hábitos dietéticos nocivos (mayor consumo de sal y de grasas saturadas) en gente procedente del medio rural, hábitos que favorecen el desarrollo de hipertensión (15).

El nivel de estudios académicos llevados a cabo por la población es muy bajo con un $66 \%$ de pacientes sin ningún tipo de estudio. Esta observación coincide con otro trabajo epidemiológico publicado recientemente, en los que se establece una relación directa entre una mayor prevalencia de HTA y escasos o nulos estudios académicos (16). La explicación se podría interpretar a la vista de que es una población anciana y de ámbito rural, población mayoritariamente carente en nuestro país de posibilidades académicas en los tiempos de su juventud, que coincidió con el período de la Guerra Civil española.

El diagnóstico de HTA fue establecido en casi todos los pacientes, en el ámbito de Atención Primaria (Médico de 


\section{TABLA I}

\begin{tabular}{|c|c|c|c|}
\hline & Edad $\geq 80$ años & Edad $\leq 80$ años & $p$ \\
\hline Hombres & 21 & 44 & \\
\hline Mujeres & 71 & 38 & $<0^{\prime} 01^{*}$ \\
\hline \multicolumn{4}{|l|}{ Estudios } \\
\hline Bachiller & $3(13 \%)$ & $4(5 \%)$ & \multirow{3}{*}{$0 ’ 864$} \\
\hline E. básico & $7(7 \%)$ & $9(11 \%)$ & \\
\hline Ninguno & $61(66 \%)$ & $72(75 \%)$ & \\
\hline Dislipemia & $25(27 \%)$ & $39(47 \%)$ & $0015^{*}$ \\
\hline Dabete & $38(41 \%)$ & $34(41 \%)$ & $0 ` 723$ \\
\hline Cardiopatía coronaria & $14(15 \%)$ & $18(22 \%)$ & $0 ’ 367$ \\
\hline Enfermedad vascular periférica & $6(6 \%)$ & $6(7 \%)$ & $0 ’ 836$ \\
\hline Hipertrofia de VI & $22(24 \%)$ & $16(19 \%)$ & 0,483 \\
\hline Afectación renal & $14(16 \%)$ & $17(20 \%)$ & 0,343 \\
\hline \multicolumn{4}{|l|}{ Evolución } \\
\hline Curación & $74(80 \%)$ & $67(82 \%)$ & \multirow{3}{*}{0,226} \\
\hline Fallecimiento & $8(8,7 \%)$ & $3(3 \%)$ & \\
\hline Traslado & $10(11 \%)$ & $12(14 \%)$ & \\
\hline Días de estancia & $11(7-18)$ & $10(5,75-14)$ & 0,126 \\
\hline
\end{tabular}

\section{Tratamiento}

\begin{tabular}{lccc} 
Diuréticos & $48(52 \%)$ & $44(53 \%)$ & 0,881 \\
IECAs & $40(43 \%)$ & $29(35 \%)$ & 0,252 \\
ARA-Il & $13(14 \%)$ & $9(11 \%)$ & 0,519 \\
Beta-bloqueantes & $4(4 \%)$ & $9(11 \%)$ & 0,099 \\
Calcioantagonistas & $22(24 \%)$ & $28(34 \%)$ & 0,141 \\
\hline Evento cardiovascular previo & $56(61 \%)$ & $42(51 \%)$ & 0,200 \\
\hline Glucemia $(\mathrm{mg} / \mathrm{dl})$ & 130,5 & 137,5 & 0,546 \\
\hline Fibrinógeno (mg/dl) & $(100,25-156)$ & $(100,75-132,75)$ & $0,022^{*}$ \\
\hline Colesterol total (mg/dl) & 422 & 486 & 0,344 \\
\hline Colesterol LDL (mg/dl) & $(364,75-527)$ & $(396,5-620,5)$ & \\
\hline Triglicéridos (mg/dl) & 178 & 187,5 & 0,303 \\
\hline Colesterol HDL (mg/dl) & $(157-200,5)$ & $(154,5-217,25)$ & $0,031^{*}$ \\
& $(73-128,-42,083$ & $123,89+-45,145$ & 0,265 \\
\hline
\end{tabular}

Familia y personal de enfermería adjunto) tal y como es de esperar. Existen de forma anecdótica algunos pacientes diagnosticados por especialistas de atención hospitalaria.

El análisis de los diferentes factores de riesgo cardiovascular en la población establece globalmente la existencia de personas de alto riesgo al coincidir en casi dos terceras partes de los casos (62\%) 4 o mas factores de riesgo. Dichos resultados esperados, por las propias características de la población estudiada, en la que se establecieron dos factores de RCV desde le principio (edad e HTA). A continuación aparecen de forma destacada la diabetes y la dislipemia, estos dos últimos factores condicionados quizás, por el estilo de vida correspondiente a una población mayoritariamente rural (15).

El porcentaje encontrado de lesiones de órgano diana y de algunos estados clínicos asociados es netamente inferior a lo esperado, en función de resultados previos de otros trabajos 
$(17,18)$, y mas sabiendo que en el grupo estudiado existían documentados eventos cardiovasculares previos (fallo cardíaco y enfermedad cerebro-vascular) en casi dos terceras partes de los casos $(60 \%)$. Encontramos una explicación a este hecho en la escasez de realización de estudios complementarios en la población estudiada, previo a su ingreso en nuestra Unidad.

Los hipertensos separados en dos grupos (según la causa del ingreso tuviese relación directa o no, con la HTA) presentan un número similar de pacientes. En el grupo de aquellos con ingreso relacionado directamente con la HTA, destaca en primer lugar el fallo cardíaco, resultados coincidentes con los datos, que establecen a la insuficiencia cardíaca como primera causa de ingreso por cardiopatía en ancianos (17). En segundo lugar está la enfermedad cerebrovascular seguida por la afectación renal. En párrafos anteriores se especificó que por las propias características del centro que no cuenta con Unidad de Cuidados Intensivos y también por las inherentes a la población estudiada, no encontramos pacientes con síndrome coronario agudo, salvo episodios anecdóticos no subsidiarios de ingreso en UCI o Unidad de Coronarias.

La mortalidad global de la serie fue del 8,6\%, más del doble de la documentada en un meta-análisis que englobó 1.670 hipertensos con edad por encima de 80 años de los recientes estudios aleatorizados (7). Esta diferencia es comprensible por el sesgo de selección: mientras nuestra población es un grupo que requiere ingreso y por tanto son pacientes con inestabilidad clínica de grado variable; la población englobada en el meta-análisis, es en líneas generales un grupo clínicamente estable. Comparando los dos grupos de nuestro estudio, observamos que en el conjunto con edad menor de 80 años, el porcentaje de mortalidad es tres veces inferior. Desde hace años es conocida que los pacientes hipertensos a mayor edad, existe mas riesgo de fallecimiento (19). En el análisis estadístico de la mortalidad en el conjunto de la población hipertensa ingresada en nuestro hospital (total 174 pacientes), los dos únicos factores con significación estadística en el grupo de los fallecidos fueron: la mayor edad y la diabetes.

La población estudiada confiesa realizar las medidas higiénico-dietéticas en siguiente orden de frecuencia: abstinencia de tabaco y alcohol, y disminución del consumo de sal. La presencia de las dos primeras está fundamentada por ser mayoritariamente mujeres las integrantes del grupo, bien sabido que son escasamente consumidoras de tabaco y alcohol. La restricción de sal es una de las recomendaciones a insistir en toda la población hipertensa, pero sobre todo en ancianos, al ser considerados hipertensos sal-sensibles. Aunque no hemos hecho control de natriuresis para confirmar si dicha aseveración era cierta entre la población estudiada, pensamos que algunos de los pacientes realmente no hacían la dieta baja/sin sal, dado que en un porcentaje nada desdeñable de casos hemos tenido que disminuir el número de fármacos al alta, tras comprobar durante su estancia hospitalaria, que con el tratamiento antihipertensivo habitual antes de su ingreso, presentaban cifras de TA en rangos bajos con clínica acompañante.

El número de pacientes que admite realizar las medidas higiénico-dietéticas es alto: 3 o más medidas en aproximadamente el $75 \%$ de la población. Pensamos que quizás por la experiencia obtenida de forma indirecta con la "dieta sin sal", dicha cifra no sea real y por tanto dicho porcentaje sea sensiblemente inferior, sobre todo en relación con las medidas dietéticas; así la menos practicada es la restricción de grasas en la dieta, en parte explicable por ser una población mayoritaria- mente del medio rural, en la que un importante componente en la alimentación son las grasas saturadas de tipo animal.

Existe una clara tendencia a la politerapia en el tratamiento antihipertensivo, explicable al ser una población de pacientes muy ancianos, además asociada a un número importante de diabéticos dentro de este grupo. Ambos factores (edad avanzada y diabetes) se relacionan en líneas generales, con un manejo terapéutico más difícil de la hipertensión, precisando asociación de diferentes fármacos para su control. En el apartado de las familias de agentes anti-hipertensivos, es clara la mayor utilización de diuréticos respecto a otros fármacos, coincidiendo con lo observado en otras series $(6,9)$. Esperábamos encontrar un mayor número de pacientes tratados con beta-bloqueantes, habida cuenta que entre los episodios previos de tipo cardiovascular, ocupa el primer lugar el fallo cardíaco. Desconocemos si este hecho se encuentra en relación con el deficiente uso en nuestro país de los beta-bloqueantes en el seno de la insuficiencia cardíaca (20), o si es debido a los efectos secundarios e importantes que este tipo de fármacos produjo en los ancianos, y que obligó a suspender su utilización.

Para un mejor control de las cifras de TA en los pacientes ingresados se modificó el número de fármacos hipertensivos en el $46 \%$ de los ingresados. Fue preciso un incremento del número de fármacos en un $24 \%$ de los casos, siendo dicho incremento muy inferior a lo descrito en otras publicaciones (21). Por el contrario en el $22 \%$ fue necesario disminuir el número de dichos fármacos por existir cifras tensionales en rangos muy bajos y con sintomatología asociada a un excesivo tratamiento antihipertensivo; esto último explicable por el mejor control tensional de los pacientes, al mantener durante su ingreso hospitalario unas medidas higiénico dietéticas adecuadas y un periocidad mantenida en el tratamiento farmacológico.

En un intento de establecer el grado de conocimiento que sobre la enfermedad hipertensiva tenían los pacientes incluidos en nuestro estudio, se pasó una encuesta sencilla, cuyas conclusiones a nuestro entender son en cierto modo paradójicas. Con las contestaciones a la primera pregunta se establece, que los hipertensos estudiados tienen un conocimiento exacto sobre las graves consecuencias para la salud que la HTA produce, entendidas dichas consecuencias de forma genérica como: problemas cerebrales, cardíacos, y muerte. A pesar de lo anteriormente referido sólo un $12 \%$ de los encuestados podía decir de forma exacta un valor límite de HTA. Estos datos concuerdan con los existentes en trabajos similares, incidiendo en la necesidad de administrar información a los pacientes con el fin de conseguir una mayor implicación del afectado en su proceso patológico, bien sea desde Atención Primaria, o desde Atención Especializada (22).

El análisis estadístico realizado para comparar entre las dos poblaciones de hipertensos, separados por el límite de edad (Tabla I), únicamente encuentra significación estadística en el grupo de muy ancianos el sexo, existe un claro predominio de mujeres en este grupo, por razones ya aclaradas en párrafos anteriores.

\section{CONCLUSIONES}

Los hipertensos muy ancianos ingresados en nuestro centro, son fundamentalmente mujeres, de procedencia rural y sin estudios académicos. Dicho ingreso es atribuible directamente a la HTA en la mitad de los casos. Son una población 
de alto riesgo cardiovascular, con eventos previos fundamentalmente cardíacos y cerebro-vasculares. La afectación de órganos diana documentada previa a su ingreso, es escasa. Confiesan realizar de forma importante las medidas higiénico-dietéticas recomendadas. Precisan politerapia, siendo los diuréticos los fármacos mas utilizados para la HTA. Como era de esperar la mortalidad en este grupo es muy alta. Las personas que conforman este grupo conocen las complicaciones derivadas de un mal control de su patología hipertensiva, aunque ignoran casi totalmente las cifras que la definen.

\section{Bibliografía}

1. Kearney PM,Whelton M, Reynolds K, Whelton PK, He J. Worldwide prevalence of hypertension: a systematic review. J Hypertension 2004; 22: 11-19.

2. Fagard RH. Epidemiology of hypertension in the elderly. Am J Geriatr Cardiol 2002; 11: 23-28.

3. Forette B. Hypertension in very old subjects. Clin Exp Hypertens 1999; 21: 917-925.

4. Stokes J III. Kannel WB, Wolf PA, D’Agostino RB, Cupples LA. Blood pressure as a risk factor for cardiovascular disease: the Framingham study-30 years of follow-up. Hypertension 1989; 31: I13-I18.

5. Elliott WJ. Management of hypertension in the very elderly patient. Hypertension 2004; 44: 800-804.

6. Boshuizen HC, Izaks GJ, Van Buuren S, Ligthart GJ. Blood pressure and mortality in elderly people aged 85 and older. Community based study. BMJ 1998; 316: 1780-1784

7. Gueyffier F, Bulpitt C, Boissel JP, Schrom E, Ekbom T, Fagard R, et al. Antihypertensive drugs in very old people: a subgroup metaanalysis of randomized controlled trials. INDANA Group. Lancet 1999; 353: 793796.

8. Trenkwalder P, Hendricks P, Schöniger R, Rossberg J, Lydtin H, Hense HW. Hypertension as a risk factor for cardiovascular morbidity and mortality in an elderly german population. The prospective STEPHY II Study. Eur Heart J 1999; 20: 1752-1756.

9. Van Rossum CTM, Van de Mheen HV, Witteman JCM, Hofman A, Mackenbach JP, Grobbee DE. Prevalence, Treatment and control of hypertension by sociodemographic factors among the Dutch Elderly. Hypertension 2000; 35: 814-821.

10. Bulpitt CJ, Beckett NS, Cooke J, Dumitrascu DL, Gil-Extremera B, Nachev C, Nunes M, Peters R, Staessen JA, Thijs L. Hypertension in the Very Elderly Trial Working Group. Results of the pilot study for the hypertension in the very elderly trial. J Hypertens 2003; 21: 24092417.

11. 2003 European Society of Hypertension-European Society of Cardiology guidelines for the management of arterial hypertension. European Society of Hypertension-European Society of Cardiology Guidelines Committee. J Hypertens 2003; 21: 1011-53.
12. Tratamiento de la hipertensión: por qué, cuándo, hasta dónde. En: Hipertensión Clínica. Kaplan NM. Lippincott Willians \& Wilkins 2003: 176205.

13. Wilmanska J, Biene B, Wojszel B. Hypertension in the advanced old aged: prevalence and treatment in the comparative urban and rural survey. Przegl Lek 2002; 59: 252-255.

14. Prospective Studies Collaborative. Age-specific relevance of usual blood pressure to vascular mortality: a metaanalysis of individual data for one million adults in 61 prospective studies. Lancet 2002; 360: 19031913.

15. Costa Matos A, Marice Ladeira A. Assessment of cardiovasculra risk factors in a rural community in the brzilian state of Bahia. Arq Bras Cardiol 2003; 81: 297-302.

16. Psaltopoulou T, Orfanos P, Naska A, Lenas D, Trichopoulos D, Trichopoulou A. Prevalence, awareness, treatment and control of hypertension in a general population sample of 26.913 adults in the Greek EPIC study. Int J Epidemiol 2004; 33: 1345-1352.

17. Sáez T, Suárez C, Blanco F, Gabriel R. Epidemiología de las enfermedades cardiovasculares en la población anciana española. Rev Esp Cardiol 1998; 51: 864-873.

18. O'Sullivan C, Duggan J, Lyons S, Thornton, Lee M, O'Brien E. Hypertensive target-organ damage in the very elderly. Hypertension 2003; 42: 130-135.

19. Siegel D, Kuller L, Lazarus NB, Black D, Feigal D, Hughes C, et al. Predictors of cardiovascular events and mortality in the systolic hypertension in the elderly program pilot project. Am J Epidemiol 1987; 126 : 385-399.

20. Salvador MJ, Sebaoum A, Sonntag F, Blanch P, Silber S, Aznar J, et al., Council for Cardiology Practice de la Sociedad Europea de Cardiología. European Study of ambulatory management of heart failure by cardiologist. Rev Esp Cardiol 2004; 57: 136-138.

21. Onder G, Gambassi G, Sgadari A, Williamson JD, Cesari M, Landi F, et al. Italian group of Pharmacoepidemiology in the Elderly Study Investigators. Pharmacotherapy 2003; 23: 240-247.

22. Roca B, Nadal E, Rovira RE, Valls S, Lapuebla C, Lloria N. Usefulness of hypertension education program. South Med J 2003; 96: 1135-1137. 